\title{
Neoplasm of uncertain behaviour of the breast-a retrospective study in a breast unit
}

\author{
Xavier de Sousa ${ }^{1}$, Pedro Santos Ferreira ${ }^{2}$, Luís Branco${ }^{2}$, Jorge Simões ${ }^{3}$, Matilde Gonçalves ${ }^{4}$, Manuel Vítor Rigueira ${ }^{2}$ and Luís Cortez ${ }^{1}$ \\ ${ }^{1}$ General Surgery Department, Setúbal Hospital Centre, São Bernardo Hospital, 2910-446 Setúbal, Portugal \\ ${ }^{2}$ Breast Unit, General Surgery Department, Setúbal Hospital Centre, São Bernardo Hospital, 2910-446 Setúbal, Portugal \\ ${ }^{3}$ Breast Unit, Obstetrics and Gynaecology Department, Setúbal Hospital Centre, São Bernardo Hospital, 2910-446 Setúbal, Portugal \\ ${ }^{4}$ Pathology Department, Setúbal Hospital Centre, São Bernardo Hospital, 2910-446 Setúbal, Portugal
}

Correspondence to: Xavier de Sousa. Email: xavierpdesousa@gmail.com

\section{Abstract}

Introduction: Breast lesions include a heterogeneous group of entities with variable clinical behaviour and morphological presentation, mostly classified as benign or malignant, with predictable behaviour. However, there are lesions with clinical, breast imaging and/or biopsy characteristics that do not clarify their nature. These lesions have an uncertain behaviour regarding their malignant potential at diagnosis.

We intend to relate the preoperative diagnosis of neoplasm of uncertain behaviour of the breast (NUnBB) regarding the core needle biopsy and the histological result after excisional biopsy.

Methods: This is a retrospective study of patients submitted to local excision of breast lesions with a perioperative diagnosis of NUnBB, classified as 2383 at 'International Statistical Classification of Diseases and Related Health Problems' (ICD 9), between January 2007 and October 2016 in a breast unit.

Results: Ninety-two cases with the diagnosis of NUnBB were analysed: 91 females with a mean age of $59 \pm 14$ years. All were submitted to local excision of breast lesion as ambulatory surgery with the following histology: $64 \%$ benign, $3 \%$ malignant potential and $33 \%$ malignant. Of those who presented malignant results, $69 \%$ underwent a surgical re-intervention for local control of the disease.

Published: 31/05/2018

Received: 19/12/2017

ecancer 2018, 12:839 https://doi.org/10.3332/ecancer.2018.839

Copyright: (C) the authors; licensee ecancermedicalscience. This is an Open Access article distributed under the terms of the Creative Commons Attribution License (http://creativecommons.org/licenses/by/3.0), which permits unrestricted use, distribution, and reproduction in any medium, provided the original work is properly cited. 
Discussion: Regarding the considerable number of malignant lesions at final histology and the high percentage of which are re-operated, NUnBB should be treated with the same priority as a confirmed malignant neoplasm and whenever possible, using the most appropriate surgical technique.

Keywords: neoplasm of uncertain behaviour of the breast, malignant potential, core needle biopsy, excisional biopsy

\section{Introduction}

Cells of the mammary gland present a high degree of phenotypic plasticity, which is reflected in the diversity of breast lesions, normally classified as benign or malignant [1]. However, some lesions include a heterogeneous group of entities, with a variable histology and clinical presentation [2] that does not clarify their nature at the moment of diagnosis. They consist of a broad spectrum of lesions with variable risk of progression, thus with an uncertain behaviour regarding their malignant potential [1, 3].

Borderline lesions with an uncertain behaviour, diagnosed with core needle biopsy (CNB) include atypical ductal hyperplasia (ADH), lobular neoplasia, papillary lesions (PL), complex sclerosing lesions (CSLs) and fibroepithelial lesions [3-13].

ADH is a lesion that exhibits a spectrum from mild atypical changes to low grade ductal carcinoma in situ (DCIS) [9, 12-14]. However, it does not have any recognisable symptoms or breast imaging abnormalities [6]. Usually, biopsies are done due to microcalcifications in mammograms $[9,15]$. The amount of tissue available in a biopsy is insufficient for a definitive diagnosis. Up to $40 \%$ of ADH is upgraded to DCIS or invasive carcinoma [12,13], having ADH means an increased risk of developing breast cancer in the future [9, 15]. Although it is not certain whether ADH is a marker of breast cancer risk or a precursor lesion, these lesions should be excised for definitive characterisation $[9,12,13]$.

CSLs, which include radial scars, are characterised by dense stromal fibrosis with stellate proliferation that distorts the surrounding tissues. It may mimic the malignancy on radiographic exams. Malignancy may not be reliably excluded after biopsy and the lesion may be recommended for excision [9, 12-15].

PLs encompass a broad spectrum of benign, atypical and malignant neoplasms [16]. CNBs may present only with a sample of PLs, in which only a segment of benign epithelium may represent carcinoma elsewhere [12]. PLs are at a greater risk of developing breast cancer; therefore, excision is advised [13, 14].

Fibroepithelial lesions are a heterogeneous group of lesions mostly comprising fibroadenomas and phyllodes tumours [12, 15]. Both share some overlapping features; however, accurate differentiation between them may sometimes be difficult with CNB. Diagnosing phyllodes tumour with CNB may be equivocal, and when a cellular fibroepithelial lesion is difficult to classify, it should be totally excised for definitive classification [12, 13, 17].

The purpose of this study is to relate the preoperative diagnosis of neoplasm of uncertain behaviour of the breast (NUnBB) regarding the results of the CNB with the histological result after its excisional biopsy and how this would impact the patients' lives.

\section{Methods and materials}

A retrospective study was performed regarding all patients submitted to an excisional biopsy of a diagnosis of NUnBB (ICD 9 2838), between January 2007 and October 2016, in a breast unit. Hospital board approval was obtained and written informed consent of the patients was not required. All data were obtained consulting the patients' hospital file. The variables considered were age and gender, result of CNB, histological result of excisional biopsy of the breast lesion and follow-up. Patients who did not present all this information were excluded.

All patients included had CNB showing a NUnBB, a discordant breast image result or a breast lesion with a suspicious clinical behaviour.

All CNB results were classified as benign, as breast lesions with indication for excision (BLIE) or as insufficient and were related with the histological result of the excised breast lesion. The excised breast lesions were classified as benign, as with malignant potential (WMP) or as malignant. 
CNB classified as benign was fibroepithelial lesions and cysts, all with high breast imaging/clinical suspicion of malignancy; as BLIE was $\mathrm{CSL} /$ radial scars, PL and ADH most with breast imaging/clinical suspicion of malignancy; and as insufficient were breast lesions with breast imaging/clinical suspicion of malignancy but in which CNB was inconclusive.

Histological result of the excisional biopsy classified as benign were sclerosing lesions, intraductal papillomas, usual ductal hyperplasias, fibroadenomas and cysts; as WMP was ADH; and as malignant was in situ and invasive carcinomas.

Follow-up after excisional biopsy was in Senology consultation. According to the definitive histological result of malignant breast lesions, patient destination was oncology consultation or surgical re-intervention.

Statistical analysis was performed using the IBM SPSS programme, version 21. A descriptive analysis of the variables was performed. Categorical variables are presented as frequencies and percentages, and numeric variables, when shown to have normal distribution, are presented with mean and standard deviation. We tested the difference between the mean of the ages of the three groups (benign, WMP and malignant) using one-way ANOVA. Statistically significant results were shown with $p$-values $<0.05$.

\section{Results}

During the mentioned period, there were a total of 123 patients with the preoperative diagnosis of NUnBB. Thirty-one were excluded for not having all the information required for the study. A total of 92 patients were included in this study.

Of the 92 patients, 91 (99\%) were female. The mean age was $59 \pm 14$ years with age range between 25 and 93 years (Table 1).

Breast imaging results demonstrated that $72 \%$ of all patients presented with breast imaging-reporting and data system (BI-RADS) higher than $3 \%$ and $11 \%$ did not have a BI-RADS classification on the report (Table 2). The breast images without report showed either highly suspicious breast nodes, distortion of the breast parenchyma or irregular microcalcifications.

Table 1. Population, $\mathbf{N}=92$.

\begin{tabular}{|l|c|}
\hline Gender (\%) & 1 \\
\hline Male & 99 \\
\hline Female & $59 \pm 14[25-93]$ \\
\hline Age (years) & (y) \\
\hline
\end{tabular}

Table 2. Breast imaging results, $N=92$.

\begin{tabular}{|l|l|l|}
\hline \multicolumn{1}{|c|}{ BI-RADS- $\boldsymbol{n}$ (\%) } & \multicolumn{1}{|c|}{ CNB- $\boldsymbol{n}$} & Excisional biopsy- $\boldsymbol{n}$ \\
\hline $2-4(4)$ & $\begin{array}{l}\text { Benign-1; } \\
\text { BLIE-3 }\end{array}$ & Benign-4 \\
\hline $3-12(13)$ & $\begin{array}{l}\text { Benign-1; } \\
\text { BLIE-11 }\end{array}$ & $\begin{array}{l}\text { Benign-11; } \\
\text { Malignant potential-1 }\end{array}$ \\
\hline $4-61(66)$ & $\begin{array}{l}\text { Benign-3; } \\
\text { BLIE-49; } \\
\text { Insufficient-9 }\end{array}$ & $\begin{array}{l}\text { Benign-39; } \\
\text { Malignant potential-2; } \\
\text { Malignant-20 }\end{array}$ \\
\hline $5-5(5)$ & $\begin{array}{l}\text { BLIE-4; } \\
\text { Insufficient-1 }\end{array}$ & Malignant-5 \\
\hline U-10 (11) & $\begin{array}{l}\text { BLIE-9; } \\
\text { Insufficient-1 }\end{array}$ & $\begin{array}{l}\text { Benign-5; } \\
\text { Malignant-5 }\end{array}$ \\
\hline
\end{tabular}

BI-RADS—breast imaging-reporting and data system; CNB—core needle biopsy; BLIE-breast lesion with indication for excision; U—unknown BI-RADS

CNB results were $5 \%$ benign, $83 \%$ BLIE and $12 \%$ insufficient. 
CNB results were $5 \%$ benign, $83 \%$ BLIE and $12 \%$ insufficient (Table 3 ).

All patients were submitted to excisional biopsy of the breast lesion in ambulatory surgery.

The histological results of the excisional biopsy were: $64 \%$ benign, 3\% WMP and 33\% malignant (Table 3).

Of the patients with CNB showing BLIE, excisional biopsy of the breast lesion showed that 24 (32\%) were malignant and 29 (64\%) were benign (Table 4).

Of the patients with malignant breast lesions, CNB showed that $24(80 \%)$ were BLIE and $5(17 \%)$ were insufficient (Table 4).

Table 3. Histological results, $\mathbf{N}=\mathbf{9 2}$.

\begin{tabular}{|l|c|}
\hline CNB- $\boldsymbol{n}(\%)$ & $5(5)$ \\
\hline Benign & $76(83)$ \\
\hline BLIE & $11(12)$ \\
\hline Insufficient & $59(64)$ \\
\hline Excisional biopsy-n (\%) & $3(3)$ \\
\hline Benign & $30(33)$ \\
\hline WMP & \\
\hline Malignant & (3) \\
\hline
\end{tabular}

CNB—core needle biopsy; BLIE—breast lesion with indication for excision;

WMP—with malignant potential

Table 4. Correlation of CNB with excisional biopsy, $\mathbf{N}=92$.

\begin{tabular}{|c|c|}
\hline CNB (n) & Excisional biopsy $(n)$ \\
\hline \multicolumn{2}{|l|}{ Benign } \\
\hline Fibroepithelial lesion (4) & Fibroadenoma (4) \\
\hline Cyst (1) & Invasive papillary carcinoma (1) \\
\hline \multicolumn{2}{|l|}{ BLIE } \\
\hline $\mathrm{ADH}(19)$ & $\begin{array}{l}\text { Benign (8); } \\
\text { ADH (1); } \\
\text { DCIS (7); } \\
\text { DCIS with microinvasion (1); } \\
\text { Invasive carcinoma (2) }\end{array}$ \\
\hline CSL/radial scar (23) & $\begin{array}{l}\text { Benign (18); } \\
\text { DCIS (2); } \\
\text { Invasive Carcinoma (3) }\end{array}$ \\
\hline PL (34) & $\begin{array}{l}\text { Benign (23); } \\
\text { ADH (2); } \\
\text { DCIS (2); } \\
\text { Intracystic papillary carcinoma (1); } \\
\text { Invasive papillary carcinoma (6) }\end{array}$ \\
\hline Insufficient (12) & $\begin{array}{l}\text { Benign (6); } \\
\text { DCIS (1); } \\
\text { Cribiform invasive carcinoma (1); } \\
\text { Invasive carcinoma (3) }\end{array}$ \\
\hline
\end{tabular}

BLIE—breast lesion with indication for excision; DCIS—ductal carcinoma in situ; ADH—atypical ductal hyperplasia 
Table 5. Destination following Senology consultation, $N=30$.

\begin{tabular}{|l|c|}
\hline Oncology consultation $(\mathrm{n})$ & 30 \\
\hline Surgical re-intervention $(\mathrm{n})$ & \\
\hline SNLB & 16 \\
\hline Margin excision + SNLB & 4 \\
\hline Mastectomy + SNLB & 1 \\
\hline
\end{tabular}

SNLB—sentinel lymph node biopsy

In comparison with the result of benign breast lesion after excision, it has been demonstrated that there was a statistical significant age difference $(p=0,017)$ between the benign breast lesions after excision and the malignant breast lesions. The mean age for benign breast lesions was $56 \pm 14$ years and for malignant was $64 \pm 13$ years.

Of the breast lesions with malignant results, 21 (69\%) had to be submitted for a surgical re-intervention (Table 5).

\section{Discussion}

No clinical, imaging or biopsy characteristics have been shown to accurately identify breast lesions of uncertain behaviour [4, 5], thus not being able to identify which lesion will progress to malignancy or not [6], obliging surgeons to totally excise these lesions in order to obtain the final diagnosis.

The treatment strategy of breast lesions with uncertain malignant potential at diagnosis is not well established [4]. There is a risk of either unnecessary over or under treatment, although an accurate treatment is decisive to prevent invasive cancer [5-7].

The purpose of this study was to relate the preoperative diagnosis NUnBB regarding the results of the CNB with the histological result after its excisional biopsy and impact in the patients' lives.

Breast lesions with CNB that show diagnostic doubt in relation to their nature need to be excised for an accurate and definite histological characterisation.

There are not many studies in the literature describing NUnBB and in what way it may impact the patient's life. Although only a small number of patients were evaluated, one of the limitations of this study, the results showed that:

(a) 59 (64\%) of the patients had a benign result after excisional biopsy. These results excluded the need for any following intervention besides adequate prophylactic breast imaging according to the patient's age.

(b) $3(3 \%)$ of the lesions excised had the result of WMP, which were ADH. Given these results, tight surveillance of these patients is crucial, because of the increased risk of developing breast cancer in the future [9, 15].

(c) $30(33 \%)$ of the breast lesions excised with the diagnosis of NUnBB had a histological diagnosis of malignant neoplasm.

As is well known, breast malignancy affects older patients, with the increased risk of developing breast cancer above 60 years of age. This study showed that the patients with the result of breast malignancy belonged to group approximately a decade older than to the group whose excisional biopsy demonstrated benign breast lesions.

When facing the possibility of breast malignancy, its diagnosis should be made as soon as possible, as it may greatly impact a patient's life. Breast lesions with the preoperative diagnosis of NUnBB in our breast unit were treated with 'normal priority', with a longer response time when compared to breast lesions diagnosed as malignant before treatment. Malignant breast neoplasms are treated within 30 days after diagnosis, while NUnBB was treated between 30 and 180 days, thus delaying the treatment of a possible malignant neoplasm.

All patients with malignant neoplasm were followed in oncology consultations, but $69 \%$ of them had to be re-operated. Sentinel lymph node biopsy was performed to all of the re-operated patients, as it is indicated in the early-stage invasive breast cancer [18]. Five patients had invading margins on excisional biopsy and had to have margin excision, one of them with the need for mastectomy. 


\section{Conclusion}

Although not many breast lesions are diagnosed as NUnBB, in this study, the third one was shown to be malignant after the excisional biopsy.

These results show the need for a faster response when facing a preoperative diagnosis of NUnBB, especially in older age groups.

Bearing this in mind, it may be important to treat these patients under the following terms: surgery in an adequate time, with 'priority' scheduled treatment; appropriate surgical technique, making sure all margins are tumour free so as to avoid a surgical re-intervention and marking the area with metal clips for eventual radiotherapy.

\section{Conflicts of interest}

The authors declare that they have no conflicts of interest.

\section{Author's contributions}

Pedro Santos Ferreira has contributed equally to this article, and should also be considered first author.

\section{References}

1. Rakha EA, Badve S, and Eusebi V, etal (2016) Breast lesions of uncertain malignant nature and limited metastatic potential: proposals to improve their recognition and clinical management Histopathology 68 45-56 https://doi.org/10.1111/his.12861 PMCID: 4987288

2. Polyak K (2011) Heterogeneity in breast cancer J Clin Invest 10 3786-3788 https://doi.org/10.1172/JCl60534

3. Zhu Q, Ricci A, and Hegde P, et al (2016) Assessment of functional differences in malignant and benign breast lesions and improvements of diagnostic accuracy by using US-guided diffuse optical tomography in conjunction with conventional US Radiology 280 387-396 https://doi.org/10.1148/radiol.2016151097 PMID: 26937708 PMCID: 4976463

4. Linda A (2007) Borderline breast lesions diagnosed at core needle biopsy: can magnetic resonance mammography rule out associated malignancy? Preliminary results based on 79 surgically excised lesions Breast 17 125-131 https://doi.org/10.1016/j. breast.2007.11.002 PMID: 18083514

5. Girardi $\vee$, et al (2012) Breast lesions of uncertain malignant potential (B3 lesions) diagnosed at stereotactic guided vacuumassisted biopsy in asymptomatic population: clinical importance ECR C-0397 https://doi.org/10.1594/ecr2012/C-0397

6. Lebeau A (2010) Precancerous lesions of the breast Breast Care 5 204-206 https://doi.org/10.1159/000319451 PMID: 22590439 PMCID: $\underline{346164}$

7. Lee AH, Denley HE, and Pinder SE, et al (2003) Excision biopsy findings of patients with breast needle core biopsies reported as suspicious of malignancy (B4) or lesion of uncertain malignant potential (B3) Histopathology 42 $331-336$ https://doi. org/10.1046/j.1365-2559.2003.01582.x PMID: 12653944

8. Margenthaler JA, Duke D, and Monsees BS, et al (2006) Correlation between core biopsy and excisional biopsy in breast highrisk lesions Am J Surg 192 534-537 https://doi.org/10.1016/j.amjsurg.2006.06.003 PMID: 16978969

9. Simmons RM and Osborne MP (1999) The evaluation of high risk and pre-invasive breast lesions and the decision process for follow up and surgical intervention Surg Oncol 8 55-65 https://doi.org/10.1016/S0960-7404(99)00030-4 
10. Reynolds HE (2000) Core needle biopsy of challenging benign breast conditions: a comprehensive literature review AJR Am J Roentgenol 174 124-1250 https://doi.org/10.2214/ajr.174.5.1741245

11. Costa A (2006) Precancerous lesions of the breast Ann Oncol 17 271-273

12. Spruill $L$ (2016) Benign mimickers of malignant breast lesions Semin Diagn Pathol 33 2-12

13. Dillon MF, McDermott EW, and Hill AD, et al (2006) Predictive value of breast lesions of "uncertain malignant potential" and "suspicious for malignancy" determined by core needle biopsy Ann surg oncol 14 (2) 704-711 https://doi.org/10.1245/s10434006-9212-8 PMID: $\underline{17151788}$

14. Tennant SI, Evans A, and Hamilton LJ, et al (2008) Vacuum-assisted excision of breast lesions of uncertain malignant potential (B3) - an alternative to surgery in selected cases Breast 17 546-549 https://doi.org/10.1016/j.breast.2008.08.005 PMID: 18829318

15. Guray M and Sahin AA (2006) Benign breast diseases: classification, diagnosis, and management Oncologist $11435-449$ https://doi.org/10.1634/theoncologist.11-5-435

16. Jorns JM (2016) Papillary lesions of the breast. A practical approach to diagnosis Arch Pathol Lab Med 140 1052-1059 https://doi.org/10.5858/arpa.2016-0219-RA PMID: 27684976

17. Yang X, Kandil D, and Cosar EF, et al (2014) Fibroepithelial tumours of the breast, pathologic and immunohistochemical features and molecular mechanisms Arch Pathol Lab Med 138 25-36 https://doi.org/10.5858/arpa.2012-0443-RA PMID: 24377809

18. Chatterjee A, Serniak N, and Czerniecki BJ (2015) Sentinel lymph node biopsy in breast cancer: a work in progress Cancer $J$ 21 7-10 https://doi.org/10.1097/PPO.0000000000000090 PMID: 25611773 PMCID: 4304410 